\title{
Efeitos do isolamento social para a saúde de pessoas idosas no contexto da pandemia
}

\section{de Covid-19: um estudo de revisão integrativa}

\author{
Effects of social isolation on the health of the elderly in the context of the Covid-19 pandemic: an \\ integrative review study \\ Efectos del aislamiento social en la salud de las personas mayores en el contexto de la pandemia
}

Covid-19: un estudio de revisión integradora

Recebido: 19/03/2021 | Revisado: 26/03/2021 | Aceito: 29/03/2021 | Publicado: 09/04/2021

\author{
Gisele Kariny de Souza Davi Bezerra \\ ORCID: https://orcid.org/0000-0002-6515-3855 \\ Universidade Federal do Rio Grande do Norte, Brasil \\ E-mail: giselek@hotmail.com \\ Ana Carolina Patrício de Albuquerque Sousa \\ ORCID: https://orcid.org/0000-0001-7517-8792 \\ Universidade Federal do Rio Grande do Norte, Brasil \\ E-mail: acapas@gmail.com \\ Maria Clara Medeiros Araújo \\ ORCID: https://orcid.org/0000-0003-4810-8806 \\ Universidade Federal do Rio Grande do Norte, Brasil \\ E-mail: mclaramds@gmail.com \\ Giminiana Aline de Lucena \\ ORCID: https://orcid.org/0000-0003-4321-815X \\ Universidade Federal do Rio Grande do Norte, Brasil \\ E-mail: giminiana0109@gmail.com \\ Lucca Ferdinando Queiroz Fernandes \\ ORCID: https://orcid.org/0000-0002-0640-0588 \\ Universidade Federal do Rio Grande do Norte, Brasil \\ E-mail: queirozlucca@gmail.com \\ Pedro Henrique de Medeiros Morais \\ ORCID: https://orcid.org/0000-0003-1631-4461 \\ Universidade Federal do Rio Grande do Norte, Brasil \\ E-mail: pepe041213@gmail.com \\ Larissa de Menezes Albuquerque Coelho \\ ORCID: https://orcid.org/0000-0002-5115-5892 \\ Universidade Federal do Rio Grande do Norte, Brasil \\ E-mail: larissaalbuquerquec98@gmail.com \\ Isabelle Canuto Rabelo Barbosa \\ ORCID: https://orcid.org/0000-0001-7505-7640 \\ Universidade Federal do Rio Grande do Norte, Brasil \\ E-mail: isabellecanuto2014@hotmail.com \\ Eric Cymon do Vale Beserra \\ ORCID: https://orcid.org/0000-0003-1174-8017 \\ Universidade Federal do Rio Grande do Norte, Brasil \\ E-mail: eric.cymondovale41@gmail.com
}

\begin{abstract}
Resumo
Introdução: O processo de senescência leva ao declínio da capacidade funcional cognitiva e de respostas adaptativas que são necessárias para a execução de atividades de vida diária, levando ao maior risco de morbidade e mortalidade. Estudos comprovam que o envelhecimento pode estar associado ao isolamento social, no qual leva a diversos prejuízos para saúde física e mental. Em dezembro de 2019, o primeiro caso do novo coronavírus foi registrado e, para conter a transmissão, foram adotadas medidas sanitárias, dentre elas o isolamento social. Objetivo: Investigar os efeitos do isolamento social para a saúde física e mental em pessoas idosas durante a pandemia de COVID-19. Métodos: Trata-se de um estudo de revisão integrativa, realizado de forma sistemática elaborado a partir da pergunta norteadora "Quais os efeitos do isolamento social da pandemia de COVID-19 para a saúde física e mental em pessoas idosas?”. Resultados: Os estudos priorizaram os efeitos na saúde mental da população idosa, havendo necessidade de ampliar a investigação para a saúde física da população idosa, no contexto de isolamento social.
\end{abstract}

Palavras-chave: Isolamento social; Idoso; Saúde mental; Infecções por Coronavírus. 


\begin{abstract}
Introduction: The senescence process leads to a decline in cognitive functional capacity and adaptive responses that are necessary to perform activities of daily living, leading to a higher risk of morbidity and mortality. Studies show that aging can be associated with social isolation, which leads to several damages to physical and mental health. In December 2019, the first case of the new coronavirus was registered and, to contain the transmission, sanitary measures were taken, including social isolation. Objective: To investigate the effects of social isolation on physical and mental health in elderly community members during the COVID-19 pandemic. Methods: This is an integrative review study, carried out systematically based on the guiding question "What are the effects of the social isolation of the COVID-19 pandemic for physical and mental health in elderly people?'.Results: The studies prioritized the effects on the mental health of the elderly population, with the need to expand the investigation for the physical health of the elderly population, in the context of social isolation.
\end{abstract}

Keywords: Social isolation; Aged; Mental health; Coronavirus infections.

\begin{abstract}
Resumen
Introducción: El proceso de senescencia conduce a una disminución de la capacidad cognitiva funcional y de las respuestas adaptativas necesarias para realizar las actividades de la vida diaria, lo que conlleva un mayor riesgo de morbilidad y mortalidad. Los estudios muestran que el envejecimiento puede estar asociado con el aislamiento social, lo que conduce a varios daños a la salud física y mental. En diciembre de 2019 se registró el primer caso del nuevo coronavirus y, para contener la transmisión, se tomaron medidas sanitarias, incluido el aislamiento social. Objetivo: Investigar los efectos del aislamiento social en la salud física y mental de los ancianos de la comunidad durante la pandemia de COVID-19. Métodos: Se trata de un estudio de revisión integradora, realizado sistemáticamente a partir de la pregunta orientadora "¿Cuáles son los efectos del aislamiento social de la pandemia de COVID-19 en la salud física y mental de los ancianos?". Resultados: Los estudios priorizaron los efectos sobre la salud mental de la población anciana, con la necesidad de ampliar la investigación para la salud física de la población anciana, en el contexto del aislamiento social.
\end{abstract}

Palabras clave: Aislamiento social; Anciano; Salud mental; Infecciones por Coronavirus.

\title{
1. Introdução
}

O envelhecimento populacional tem ocorrido mundialmente, dando-se principalmente pelo aumento da expectativa de vida associado a queda da fecundidade (Da Motta \& De Aguiar, 2007; Dias Myrrha, Turra, \& Wajnman, 2017; Miranda et al., 2016; NASRI, 2008; Oliveira, 2019). Segundo dados do instituto Brasileiro de Geografia e Estatística (IBGE, 2018), espera-se que até 2058 a população brasileira acima de 65 anos seja de 25,49\%, enquanto a de jovens, de até 14 anos, será $14,72 \%$.

O envelhecimento ocorre em função do tempo de vida e trata-se de um processo fisiológico do ser humano que pode ser influenciado por diversos fatores como o gênero, classe social, poder aquisitivo, participação social, entre outros (Gorz, 2009; Hertzog, Kramer, Wilson, \& Lindenberger, 2008). Deste modo, tal processo não pode ser mensurado apenas pela idade cronológica, tendo em vista que abarca elementos mais complexos, tais como: as adversidades sociais e econômicas, que interagem entre si e que influenciam de forma variável o indivíduo com o decorrer do tempo (Schneider \& Irigaray, 2008; Sousa et al., 2014).

Com o processo de senescência ocorre o aumento da suscetibilidade a doenças que podem ter consequências incapacitantes (Kutz, Maria, Abreu, \& Salgueiro, 2016). Nele ocorrem mudanças fisiológicas que podem levar ao comprometimento das capacidades cognitivas e físicas, o que leva ao declínio da cognição, como também da mobilidade e restrição nas atividades de vida, culminando em declínios de capacidade funcional e de respostas adaptativas que são indispensáveis para a realização das atividades da vida diária (AVD’s) (Freitas et al., 2014; Gorz, 2009; Hertzog et al., 2008; Willians \& Kemper, 2010).

Estudos tem evidenciado que em grande frequência ocorre a associação entre o envelhecimento e o isolamento social (Robins, Hill, Finch, Clemson, \& Haines, 2018; Shankar, McMunn, Demakakos, Hamer, \& Steptoe, 2017; Yu, Steptoe, Niu, $\mathrm{Ku}, \&$ Chen, 2018). Este, é definido como a falta de comunicação significativa e sustentada ou interações com redes sociais (Smith, Dainty, \& Macgregor, 2018). Além disso, este fato corrobora para o aumento da suscetibilidade a perda da função cognitiva, redução da mobilidade, aumento da fadiga, perdas funcionais, problemas no sono, sentimentos de solidão e sintomas 
depressivos (Park, Jang, Lee, \& Chiriboga, 2017; RIBEIRO et al., 2019; Yu et al., 2018).

Em decorrência de sua vulnerabilidade para adoecimentos, as pessoas idosas tornam-se o principal grupo de risco para diversas doenças. Em 31 de dezembro de 2019 o primeiro caso do novo coronavírus (Severe Acute Respiratory Syndrome Coronavirus -SARS-Cov-2) foi notificado em Wuhan, na China e em 11 de março de 2020 foi declarada a pandemia mundial, responsável por milhares de mortes no mundo (Hammerschmidt \& Santana, 2020). No Brasil o primeiro caso foi anunciado em 26 de fevereiro de 2020 e o primeiro óbito em 17 de março de 2020, sendo ambos indivíduos idosos. Foi observado que quanto maior a idade do infectado pelo Corona vírus disease-19 (COVID- 19) mais elevada era o risco de mortalidade, em especial aqueles com doenças crônicas. Deste modo, as pessoas idosas se tornaram o grupo de risco e o centro de discussões sobre o cuidado e prevenção na pandemia COVID-19 (Hammerschmidt \& Santana, 2020).

Durante a pandemia foram adotadas medidas de isolamento e distanciamento social, interrupção de atividades cotidianas, além do que, diariamente eram noticiadas mortes. No momento, todos os esforços estão sendo utilizados para a prevenção da infecção por COVID-19 e suas consequências. Entretanto, é importante compreender quais as consequências negativas do isolamento social para a saúde física e mental da pessoa idosa, pois tais consequências podem ser enfrentadas por aqueles que foram ou não infectados pelo novo coronavírus.

Considerando a atual realidade e sua tendência de conduzir ao sofrimento psíquico e prejuízos físicos, este estudo tem como objetivo apresentar uma revisão da literatura das pesquisas relacionadas aos efeitos do isolamento social para a saúde física e mental em pessoas idosas durante a pandemia de COVID-19.

\section{Metodologia}

Trata-se de um estudo de revisão integrativa, realizada de forma sistemática e elaborada a partir das seis etapas: ETAPA ZERO: foi cultivado o espírito de investigação, ETAPA 1: identificou-se o tema, definiu-se o problema e pergunta clínica em formato PICOD (P: considerar a população alvo; I: considerar o interesse da intervenção ou a área de interesse; C: comparar tipos de intervenção ou grupos; O: obter resultados e considerar os efeitos a serem alcançados com a intervenção; D: desenho do estudo), ETAPA 2: busca na literatura por melhores evidencias para responder a pergunta norteadora, ETAPA 3: avaliação crítica das evidências e dos estudos pré-selecionados e selecionados, ETAPA 4: integração das evidência, ETAPA 5: discussão dos resultados baseados em evidências e apresentação dos resultados de forma clara e objetiva (Soares et al., 2010)

Como base para a pesquisa formulou-se a pergunta norteadora "Quais os efeitos do isolamento social da pandemia de COVID-19 para a saúde física e mental em pessoas idosas?".

Para busca de artigos foram utilizadas as bases de dados: Literatura Latino Americana e do Caribe em Ciências da Saúde (LILACS), Scientific Eletronic Library Online (SCIELO), Portal Periódicos Capes (para busca de dissertação e teses), Medical Literature Analysis and Retrieval System Online (MEDLINE), US National Library of Medicine (PUBMED) e Elsevier. Foram utilizados os Descritores em Ciências da Saúde (DeCS): Isolamento social (Social Isolation), idoso (Aged), envelhecimento (Aging), saúde mental (Mental Health), Infecções por Coronavírus (Coronavirus Infections ou COVID-19), sendo estes combinados com os operadores booleanos and e or.

A busca foi realizada em setembro de 2020, sendo incluídos na revisão estudos quantitativos e qualitativos, publicações a partir do ano 2000, artigos em inglês, português e espanhol (para incluir artigos da América Latina). Foram excluídos artigos narrativos, secundários, estudos de caso, guia de prática clínica, estudos observacionais, estudos repetidos e que não condiziam com a pergunta norteadora.

Os artigos pré-selecionados foram organizados em uma tabela contendo as informações e características metodológicas. Em seguida relidos os títulos, palavras-chave e resumos. Após a seleção, cada artigo foi lido na íntegra e submetido a duas análises críticas, e com base nelas montado um banco de dados com o ano de publicação, país que foi 
produzido, autores, tipo de amostra e modelo de investigação.

\section{Resultados e Discussão}

Com a busca nas bases de dados foram encontrados primariamente 4.727 artigos, sendo realizada a leitura de título e resumo culminando a pré-seleção de 66 estudos sendo excluídos 15 por estarem repetidos. Os 51 artigos pré-selecionados tiveram título e resumo relidos para classifica-los quanto aos critérios de inclusão e exclusão, 10 estudos atendiam aos critérios de inclusão e exclusão, sendo estes selecionados para análise crítica e integrados a revisão (Figura 1).

Figura 1- Fluxograma da seleção dos artigos incluídos na revisão.

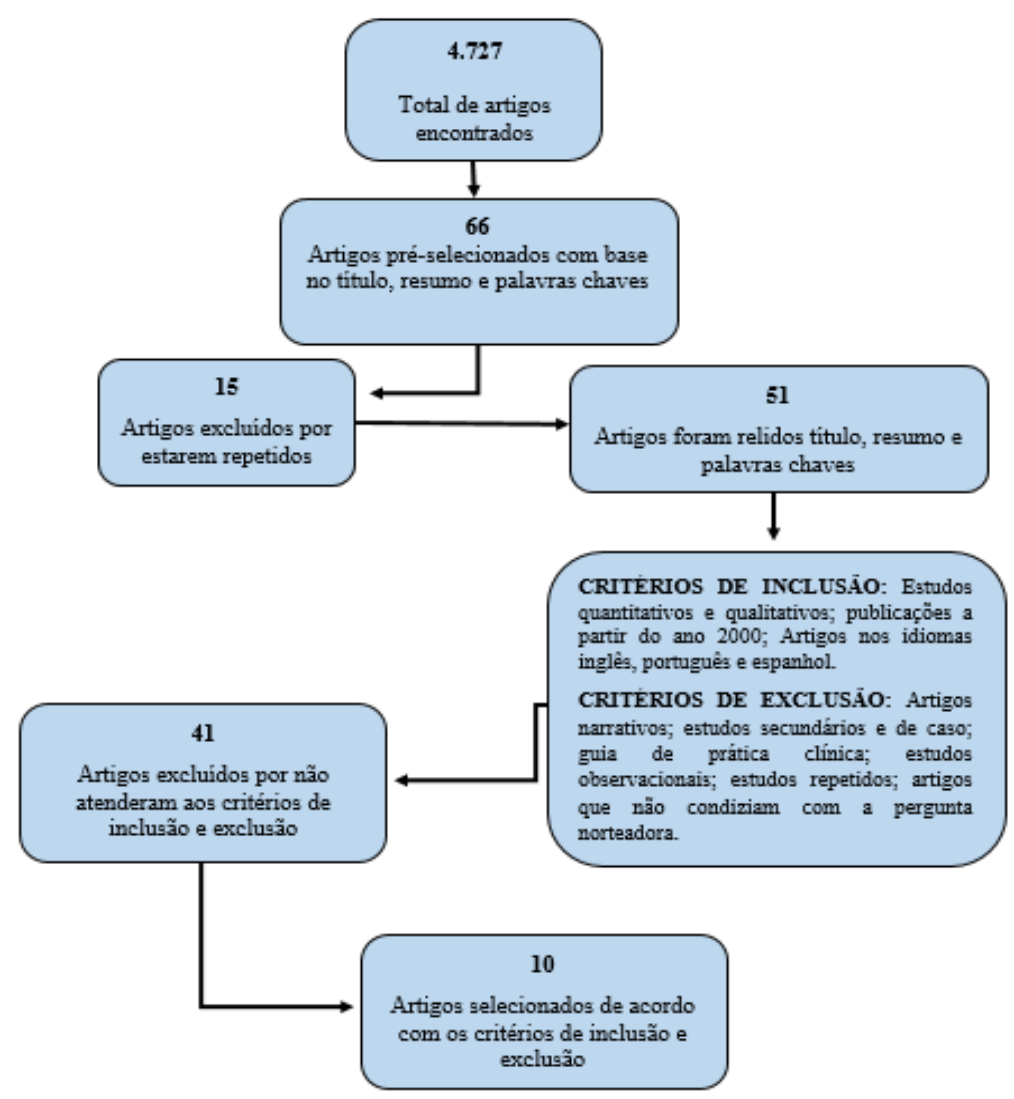

Fonte: Autores.

Durante a leitura crítica foram observadas as seguintes características dos manuscritos: ano de publicação, tipo de estudo, número amostral, metodologia, resultados e país no qual foi realizada o estudo (Tabela 1).

Os países de desenvolvimento de cada artigo foram o Brasil, Reino Unido, Suécia, China, Israel, Estados Unidos da América (EUA), Espanha e Canadá. Com relação ao tipo metodológico de cada estudo dois eram qualitativos, sete eram quantitativos e um misto (Tabela 1). 
Tabela 1- Caracterização dos estudos.

\begin{tabular}{|c|c|c|c|c|c|c|c|}
\hline Titulo & Autores & $\begin{array}{c}\text { Ano de } \\
\text { publicação }\end{array}$ & $\begin{array}{c}\text { Tipo } \\
\text { de } \\
\text { estudo }\end{array}$ & $\begin{array}{l}\text { Tamanho } \\
\text { amostral }\end{array}$ & Público Alvo & Principais Desfechos & $\begin{array}{l}\text { Local de } \\
\text { Realização } \\
\text { do Estudo }\end{array}$ \\
\hline $\begin{array}{l}\text { Saúde do idoso em tempos de } \\
\text { pandemia COVID-19 }\end{array}$ & $\begin{array}{l}\text { Hammers } \\
\text { chmidt; } \\
\text { Santana }\end{array}$ & 2020 & $\begin{array}{l}\text { Qualitati } \\
\text { vo }\end{array}$ & - & Idosos & $\begin{array}{l}\text { Necessidade de cuidado gerontológico } \\
\text { robusto, qualificado e seguro, } \\
\text { respeitado a complexidade e } \\
\text { pluralidade do envelhecimento. }\end{array}$ & Brasil \\
\hline $\begin{array}{l}\text { Associations of Social Isolation } \\
\text { with Anxiety and Depression } \\
\text { During the Early COVID-19 } \\
\text { Pandemic: A Survey of Older } \\
\text { Adults in London, UK }\end{array}$ & $\begin{array}{l}\text { Robb et } \\
\text { al. }\end{array}$ & 2020 & $\begin{array}{l}\text { Quantit } \\
\text { ativo }\end{array}$ & 7.127 & Idosos & $\begin{array}{l}\text { Mulheres, participantes mais jovens, } \\
\text { solteiros / viúvos / divorciados, sono } \\
\text { insatisfatório, sentimentos de solidão } \\
\text { e que moram sozinhos, estão mais } \\
\text { propensos e sentirem acentuação da } \\
\text { depressão e / ou ansiedade. }\end{array}$ & $\begin{array}{l}\text { Reino } \\
\text { Unido }\end{array}$ \\
\hline $\begin{array}{l}\text { Compliance to Recommendations } \\
\text { and Mental Health Consequences } \\
\text { among Elderly in Sweden during } \\
\text { the Initial Phase of the COVID-19 } \\
\text { Pandemic-A Cross Sectional } \\
\text { Online Survey. }\end{array}$ & $\begin{array}{l}\text { Gustavsso } \\
\mathrm{n} ; \quad \text { Linda } \\
\text { Beckman }\end{array}$ & 2020 & $\begin{array}{l}\text { Quantit } \\
\text { ativo }\end{array}$ & 1854 & Idosos & $\begin{array}{l}\text { Mulheres relataram mais sentimentos } \\
\text { negativos, problemas de sono, } \\
\text { dificuldade de concentração e } \\
\text { preocupação com problemas } \\
\text { econômicos do que homens. Solteiros } \\
\text { tiveram maiores prejuízos de sono. }\end{array}$ & Suécia \\
\hline 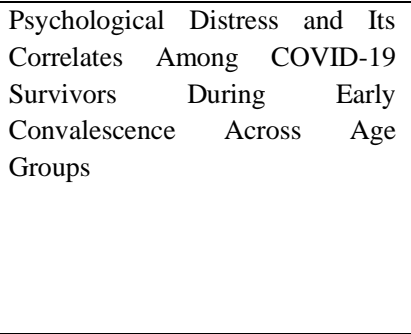 & Cai et al., & 2020 & $\begin{array}{l}\text { Quantit } \\
\text { ativo }\end{array}$ & 126 & $\begin{array}{l}\text { Sobreviventes de } \\
\text { COVID-19 }\end{array}$ & $\begin{array}{l}\text { Ser do sexo feminino e ter parentes } \\
\text { infectados foi associado aos sintomas } \\
\text { de ansiedade e depressão. Pacientes } \\
\text { mais velhos têm menor reatividade } \\
\text { emocional a infecção, menos sintomas } \\
\text { ansiosos e de resposta ao estresse } \\
\text { menos graves que os mais jovens. } \\
\text { Associou-se o bom suporte social a } \\
\text { menores escores depressivos. }\end{array}$ & China \\
\hline $\begin{array}{l}\text { COVID-19 related loneliness and } \\
\text { sleep problems in older adults: } \\
\text { Worries and resilience as potential } \\
\text { moderators }\end{array}$ & $\begin{array}{l}\text { Grossman } \\
\text { et al. }\end{array}$ & 2020 & $\begin{array}{l}\text { Quantit } \\
\text { ativo }\end{array}$ & 243 & Idosos & $\begin{array}{l}\text { Níveis mais altos de solidão } \\
\text { relacionada ao COVID-19 está } \\
\text { associado a maiores problemas de } \\
\text { sono entre os idosos. }\end{array}$ & Israel \\
\hline $\begin{array}{l}\text { COVID-19- Related Loneliness } \\
\text { and Psychiatric Symptoms Among } \\
\text { Older Adults: The Buffering Role } \\
\text { of Subjective Age }\end{array}$ & $\begin{array}{l}\text { Shrira et } \\
\text { al. }\end{array}$ & 2020 & $\begin{array}{l}\text { Quantit } \\
\text { ativo }\end{array}$ & 277 & Idosos & $\begin{array}{l}\text { Solidão foi relacionada a sintomas } \\
\text { psiquiátricos. Sentir-se mais velho } \\
\text { (maior idade subjetiva) também foi } \\
\text { relacionado a sintomas psiquiátricos. }\end{array}$ & Israel \\
\hline $\begin{array}{l}\text { Experiences of American Older } \\
\text { Adults with Pre-existing } \\
\text { Depression During the Beginnings } \\
\text { of the COVID-19 Pandemic: A } \\
\text { Multicity, Mixed-Methods Study }\end{array}$ & $\begin{array}{l}\text { Hamm et } \\
\text { al. }\end{array}$ & 2020 & Misto & 73 & Idosos & $\begin{array}{l}\text { Alguns participantes relataram } \\
\text { aumento da depressão/ansiedade, } \\
\text { qualidade de vida mais baixa e receio } \\
\text { de que a saúde mental sofresse com o } \\
\text { isolamento contínuo. }\end{array}$ & EUA \\
\hline $\begin{array}{l}\text { Mental Health in Eldery Spanish } \\
\text { People in Times of COVID-19 } \\
\text { Outbreak }\end{array}$ & $\begin{array}{l}\text { Fernández } \\
\text { et al. }\end{array}$ & 2020 & $\begin{array}{l}\text { Quantit } \\
\text { ativo }\end{array}$ & 1639 & $\begin{array}{l}\text { Idosos e Pessoas } \\
\text { mais jovens }\end{array}$ & $\begin{array}{l}\text { A limitação do engajamento social } \\
\text { entre os idosos, pela dificuldade de } \\
\text { lidar com as tecnologias digitais. Os } \\
\text { idosos apresentaram maior resiliência } \\
\text { do que pessoas mais jovens. }\end{array}$ & spanha \\
\hline $\begin{array}{l}\text { Reactions to COVID-19, } \\
\text { information and technology use, } \\
\text { and social connectedness among } \\
\text { older adults with pre-frailty and } \\
\text { frailty }\end{array}$ & Chen et al. & 2020 & $\begin{array}{l}\text { Qualita } \\
\text { tivo }\end{array}$ & 10 & Idosos & $\begin{array}{l}\text { Os participantes relataram ter } \\
\text { experimentado estresse, ansiedade e } \\
\text { preocupação devido ao COVID-19.A } \\
\text { sensação de isolamento se deu pelo } \\
\text { menor contato com seus familiares. }\end{array}$ & EUA \\
\hline $\begin{array}{l}\text { The mental health benefits of } \\
\text { physical activity in older adults } \\
\text { survive the COVID-19 pandemic }\end{array}$ & $\begin{array}{l}\text { Callow et } \\
\text { al. }\end{array}$ & 2020 & $\begin{array}{l}\text { Quantit } \\
\text { ativo }\end{array}$ & 1046 & Idosos & $\begin{array}{l}\text { A prática de exercício físico regular } \\
\text { reduz a sintomatologia depressiva. }\end{array}$ & $\begin{array}{l}\text { EUA e } \\
\text { Canadá }\end{array}$ \\
\hline
\end{tabular}

Fonte: Autores.

Os artigos selecionados abordaram dois temas principais: a) saúde mental no envelhecimento e b) isolamento social durante a pandemia de COVID-19. E em sua maioria, a pesquisa foi realizada de forma online.

Os principais achados dos estudos sobre isolamento social de idosos devido a pandemia de COVID-19 foram: mulheres estão mais propensas a sintomas de sofrimento mental, a má qualidade do sono pode levar a maior prejuízo na saúde 
psíquica, o sentimento de solidão leva a maior predisposição a sintomas de ansiedade/depressão e que o apoio social e familiar são excelentes formas de prevenção.

Estudos apontaram que durante a pandemia de COVID-19 o sexo feminino estava mais propenso a sintomas depressivos, sentimentos negativos e sentir-se deprimido (Cai et al., 2020; Gustavsson \& Beckman, 2020; Robb et al., 2020). Tais achados podem ser justificados por estudos anteriores a pandemia, tais demonstram que as mulheres tendem a ser mais solitárias e consideram as interações com amigos ou família importantes (Feng \& Astell-Burt, 2016; Gale, Westbury, \& Cooper, 2018).

$\mathrm{Na}$ literatura o isolamento social já vem sendo apontado como fator para pior qualidade de sono (Yu et al., 2018). Corroborando com os estudos que avaliaram o sono durante o distanciamento social em decorrência da pandemia, além disso, problemas no sono foram associados ao maior sentimento de solidão e por conseguintes sintomas psiquiátricos (Grossman, Hoffman, Palgi, \& Shrira, 2021; Gustavsson \& Beckman, 2020; Robb et al., 2020; Shrira, Hoffman, Bodner, \& Palgi, 2020). Destacam-se como principais queixas de pessoas idosas durante a pandemia o aumento da depressão, ansiedade, estresse, preocupação e redução da qualidade de vida (Hamm et al., 2020; Chen et al., 2020).

Há estudos que mostram que o apoio familiar tem importante papel para prevenção de sofrimento mental e que pessoas idosas com maior suporte apresentam menores escores de sintomas depressivos (Cai et al., 2020; Chen et al., 2020). A prática regular de atividade física também tem se configurado como fator de proteção para sintomatologia depressiva, seja pelos efeitos fisiológicos ou por proporcionar sociabilidade. (Callow et al., 2020). Entretanto, o distanciamento social imposto pela pandemia da COVID-19 exigiu que as pessoas deixassem de praticar atividade física em ambientes públicos (Gustavsson \& Beckman, 2020) limitando o convívio social e, podendo trazer prejuízos a médio e longo prazo para a capacidade física das pessoas idosas. Outro achado interessante que chamou a atenção nesta revisão foi o do estudo de Fernández et al., 2020, o qual relatou que durante a pandemia de COVID-19, os idosos apresentaram maior limitação no engajamento social devido a terem menos hábito de usar as tecnologias digitais, entretanto apresentam maior capacidade de resiliência quando comparados a pessoas mais jovens, o autor aponta que isso pode se dar por terem experienciado mais dificuldade anteriores (GarcíaFernández, Romero-Ferreiro, López-Roldán, Padilla, \& Rodriguez-Jimenez, 2020). Esse achado também é confirmado por estudos que relatam a baixa escolaridade da população idosa brasileira e, portanto, a dificuldade de acesso a recursos tecnológicos (Alvarenga, Delfino, Silva, Yassuda, \& Cachioni, 2018; Carvalho, Arantes, \& Cintra, 2016; Hammerschmidt \& Santana, 2020)

Hammerschmidt e Santana, 2020, também abordaram em seu manuscrito uma reflexão crítica sobre aspectos relacionados à saúde dos idosos nos tempos de pandemia de COVID-19, apontando para a diversidade e complexidade do envelhecimento humano; e defendendo que o isolamento social não deve constituir como um abandono familiar e/ou governamental, mas reforçar o cuidado gerontológico para evitar as síndromes geriátricas que envolvem o medo de morrer. (Hammerschmidt \& Santana, 2020).

\section{Considerações Finais}

O presente estudo teve como objetivo principal relatar as consequências de isolamento social para a saúde física e mental de pessoas idosas. Os estudos priorizaram os efeitos na saúde mental da população idosa, que tem se constituído como o impacto imediato para este público na pandemia. Os estudos apontaram para a limitação de atividade e restrição de participação que podem deteriorar a função física de pessoas idosas, em médio e longo prazo. A partir dessa revisão bibliográfica foi possível observar que boa parte da literatura atual se restringe a apenas uma dimensão do ser humano. Deste modo, faz-se mister que haja a ampliação do olhar do que se compreende como saúde das pessoas idosas e a influência do psíquico sobre o físico e vice-versa. 
Não foram encontrados artigos que tratassem dos prejuízos físicos para senescentes devido ao isolamento social durante a pandemia, isso pode ser justificado pelo fato das normas sanitárias orientarem quanto ao distanciamento social, o que inviabilizaria uma avaliação física, enquanto pesquisas de saúde mental puderam ser realizadas de forma virtual, por meio de questionário online. Deste modo, sugere-se que pesquisas sejam realizadas para a investigação da função física na pessoa idosa no cenário pós-pandemia; bem como a necessidade de estudos longitudinais que mensurem a sarcopenia em idosos durante a fase de isolamento social.

Foi observado que a família e o suporte social têm papéis importantes para a redução de síndromes geriátricas e de sofrimento psíquico, reforçando a necessidade da criação de estratégias que visem a estruturação de uma rede de apoio bem articulada e um acompanhamento gerontológico bem estruturado.

Tais achados servem como apoio para políticas públicas de saúde e transformações biopsicossociais para idosos na pandemia e seus desdobramentos em médio e longo prazo e também para ações em saúde em outras pandemias.

Por fim, diante dos achados, destaca-se a necessidade do desenvolvimento de mais pesquisas relacionadas com o tema desta revisão, além da criação de estratégias para lidar com momentos de calamidade pública baseadas no momento presente vislumbrando necessidades futuras.

\section{Financiamento}

Apoio financeiro: não houve financiamento.

\section{Conflitos de interesses}

Não houveram conflitos de interesses dos pesquisadores.

\section{Referências}

Alvarenga, G. M. de O., Delfino, L. L., Silva, L. D. S. V., Yassuda, M. S., \& Cachioni, M. (2018). Idosos E Inclusão Digital Com Tablet-Pc: Uma Revisão Sistemática Da Literatura. Estudos Interdisciplinares Sobre o Envelhecimento, 23(1).

Cai, X., Hu, X., Ekumi, I. O., Wang, J., An, Y., Li, Z., \& Yuan, B. (2020). Psychological Distress and Its Correlates Among COVID-19 Survivors During Early Convalescence Across Age Groups. American Journal of Geriatric Psychiatry, Vol. 28, pp. 1030-1039.

Callow, D. D., Arnold-Nedimala, N. A., Jordan, L. S., Pena, G. S., Won, J., Woodard, J. L., \& Smith, J. C. (2020). The Mental Health Benefits of Physical Activity in Older Adults Survive the COVID-19 Pandemic. American Journal of Geriatric Psychiatry, Vol. 28, pp. 1046-1057.

Carvalho, E., Arantes, R. C., \& Cintra, A. S. R. (2016). The inclusion of elderly persons from the Instituto Henrique da Silva Semente (IHESS) in Indaiatuba, São Paulo, in the digital age: physio-gerontological contributions. Revista Brasileira de Geriatria e Gerontologia, 19(4), 567-575.

Chen, AT, Ge, S., Cho, S., Teng, AK, Chu, F., Demiris, G., \& Zaslavsky, O. (2021). Reações ao COVID-19, uso de informação e tecnologia e conexão social entre idosos com pré-fragilidade e fragilidade. Enfermagem geriátrica (New York, NY), 42 (1), 188-195.

Da Motta, L. B., \& De Aguiar, A. C. (2007). Novas competências profissionais em saúde e o envelhecimento populacional brasileiro: Integralidade, interdisciplinaridade e intersetorialidade. Ciencia e Saude Coletiva, 12(2), 363-372.

Dias Myrrha, L. J., Turra, C. M., \& Wajnman, S. (2017). A contribuição dos nascimentos e óbitos para o envelhecimento populacional no Brasil, 1950 a 2100. Revista Latinoamericana de Población, 11(20), 37-54.

Feng, X., \& Astell-Burt, T. (2016). What types of social interactions reduce the risk of psychological distress? Fixed effects longitudinal analysis of a cohort of 30,271 middle-to-older aged Australians. Journal of Affective Disorders, 204, 99-102.

Freitas, E. V., Woollacott, M., Shumway-Cook, A., Mochizuki, G., Boe, S. G., Marlin, A., ... Callisaya, M. L. (2014). The role of cognitive impairment in fall risk among older adults: A systematic review and meta-analysis. Archives of Neurology, 63(1), 1763-1769.

Gale, C. R., Westbury, L., \& Cooper, C. (2018). Social isolation and loneliness as risk factors for the progression of frailty: The English Longitudinal Study of Ageing. Age and Ageing, 47(3), 392-397.

García-Fernández, L., Romero-Ferreiro, V., López-Roldán, P. D., Padilla, S., \& Rodriguez-Jimenez, R. (2020). Mental Health in Elderly Spanish People in Times of COVID-19 Outbreak. American Journal of Geriatric Psychiatry, Vol. 28, pp. 1040-1045.

Gorz, A. (2009). O envelhecimento. Tempo Social, 21(1), 15-34. 
Grossman, E. S., Hoffman, Y. S. G., Palgi, Y., \& Shrira, A. (2021). COVID-19 related loneliness and sleep problems in older adults: Worries and resilience as potential moderators. Personality and Individual Differences, Vol. 168.

Gustavsson, J., \& Beckman, L. (2020). Compliance to recommendations and mental health consequences among elderly in sweden during the initial phase of the covid-19 pandemic - a cross sectional online survey. International Journal of Environmental Research and Public Health, 17(15), 1-10.

Hamm, M. E., Brown, P. J., Karp, J. F., Lenard, E., Cameron, F., Dawdani, A., ... Lenze, E. J. (2020). Experiences of American Older Adults with Preexisting Depression During the Beginnings of the COVID-19 Pandemic: A Multicity, Mixed-Methods Study. American Journal of Geriatric Psychiatry, Vol. 28, pp. 924-932.

Hammerschmidt, K. S. de A., \& Santana, R. F. (2020). Saúde do idoso em tempos de pandemia COVID-19. Cogitare Enfermagem, Vol. 25.

Hertzog, C., Kramer, A. F., Wilson, R. S., \& Lindenberger, U. (2008). Enrichment Effects on Adult Cognitive Development. Psychological Science in the Public Interest, 9(1), 1-65.

Kutz, N. A., Maria, M., Abreu, H. De, \& Salgueiro, D. O. (2016). Relações entre equilíbrio, força muscular, mobilidade funcional, medo de cair e estado nutricional de idosos da comunidade. Revista Kairós: Gerontologia, 19(2), 147-165.

Miranda, D., Morais, G., Mendes, G., Cruz, A., Silva, A., \& Lucia, A. (2016). O envelhecimento populacional brasileiro- desafios e consequências. Revista Brasileira de Geriatria e Gerontologia, 19(3), 507-519.

Nasri, F. (2008). Demografi a e epidemiologia do envelhecimento O envelhecimento populacional no Brasil The aging population in Brazil. Einstein, 6(2), 46.

Oliveira, A. S. (2019). Transição Demográfica, Transição Epidemiológica E Envelhecimento Populacional No Brasil. Hygeia - Revista Brasileira de Geografia Médica e Da Saúde, 15(32), 69-79.

Park, N. S., Jang, Y., Lee, B. S., \& Chiriboga, D. A. (2017). The relation between living alone and depressive symptoms in older Korean Americans: do feelings of loneliness mediate? Aging and Mental Health, 21(3), 304-312.

Ribeiro, I. A., Lima, L. R. De, Vople, C. R. G., Funghetto, S. S., Rehem, T. C. M. S. B., \& Stival, M. M. (2019). Síndrome do idoso frágil em idosos com doenças crônicas na Atenção Primária. Rev Esc Enferm USP, 53(03449), 1-9.

Robb, C. E., de Jager, C. A., Ahmadi-Abhari, S., Giannakopoulou, P., Udeh-Momoh, C., McKeand, J., ... Middleton, L. (2020). Associations of Social Isolation with Anxiety and Depression During the Early COVID-19 Pandemic: A Survey of Older Adults in London, UK. Frontiers in Psychiatry, 11(September), 1-12.

Robins, L. M., Hill, K. D., Finch, C. F., Clemson, L., \& Haines, T. (2018). The association between physical activity and social isolation in communitydwelling older adults. Aging and Mental Health, 22(2), 175-182.

Schneider, R. H., \& Irigaray, T. Q. (2008). O envelhecimento na atualidade: aspectos cronológicos, biológicos, psicológicos e sociais. Estudos de Psicologia (Campinas), 25(4), 585-593.

Shankar, A., McMunn, A., Demakakos, P., Hamer, M., \& Steptoe, A. (2017). Social isolation and loneliness: Prospective associations with functional status in older adults. Health Psychology, 36(2), 179-187.

Shrira, A., Hoffman, Y., Bodner, E., \& Palgi, Y. (2020). COVID-19-Related Loneliness and Psychiatric Symptoms Among Older Adults: The Buffering Role of Subjective Age. American Journal of Geriatric Psychiatry, Vol. 28, pp. 1200-1204.

Smith, T. O., Dainty, J. R., \& Macgregor, A. (2018). Trajectory of social isolation following hip fracture: An analysis of the English Longitudinal Study of Ageing (ELSA) cohort. Age and Ageing, 47(1), 107-112.

Soares, C. B., Hoga, L. A., Peduzzi, M., Sangaleti, C., Yonekura, T., Silva, D. R. A. D., ... Trevizan, M. A. (2010). Revisão Integrativa versus Revisão Sistemática. Reme: Revista Mineira de Enfermagem, 8(1), 102-106.

Sousa, A. C. P. D. A., Guerra, R. O., Tu, M. T., Phillips, S. P., Guralnik, J. M., \& Zunzunegui, M. V. (2014). Lifecourse adversity and physical performance across countries among men and women aged 65-74. PLoS ONE, 9(8).

Willians, K., \& Kemper, S. (2010). Exploring interventions to reduce cognitive decline in aging. Journal of Psychosocial Nursing \& Mental Health Services, 48(913), 42-51.

Yu, B., Steptoe, A., Niu, K., Ku, P. W., \& Chen, L. J. (2018). Prospective associations of social isolation and loneliness with poor sleep quality in older adults. Quality of Life Research, 27(3), 683-691. 